[15] M. Grant, S. Boyd, and Y. Y. Ye, CVX: MATLAB Software for Disciplined Convex Programming, 2006. [Online]. Available: http://www. stanford.edu/ boyd/cvx/

[16] A. Pascual-Iserte, D. P. Palomar, A. I. Prez-Neira, and M. A. Lagunas, "A robust maximin approach for MIMO communications with imperfect channel state information based on convex optimization," IEEE Trans. Signal Process., vol. 54, no. 1, pp. 346-360, Jan. 2006.

\section{Precoded Sphere-Packing-Aided Bit-Interleaved Differential Space-Time Coded Modulation Using Iterative Decoding}

\author{
N. Wu, O. Alamri, S. X. Ng, and L. Hanzo
}

\begin{abstract}
In this treatise, we investigate a precoded and sphere packing (SP)-aided bit-interleaved differential space-time coded modulation scheme using iterative decoding. The amalgamated design and interworking of the SP, differential encoding, and iteratively detected bit-interleaved schemes are investigated. A rate-1 precoder is integrated into the system, where iterative decoding is carried out between the precoder and an outer channel code. We identify the optimal SP mapping and investigate the convergence behavior of this concatenated scheme with the aid of EXtrinsic Information Transfer (EXIT) charts. Furthermore, we consider various rate-1 precoders having different memory sizes and demonstrate that the memory-1 precoder performs best when an $\operatorname{RSC}(2,1,5)$ is employed as the outer code, creating an open EXIT chart convergence tunnel at $E_{b} / N_{0}=6 \mathrm{~dB}$. On the other hand, the memory-3 precoder is more suitable for the proposed system employing irregular convolutional codes, resulting in an infinitesimally low bit error rate at $E_{b} / N_{0}=5.5 \mathrm{~dB}$.
\end{abstract}

Index Terms-Differential space-time block code (STBC), EXtrinsic Information Transfer (EXIT) charts, iterative detection, sphere packing.

\section{INTRODUCTION}

The use of multiple antennas at the transmitter and receiver of a wireless communication system offers both high diversity gains and high data rates. Bit-Interleaved Space-Time Coded Modulation using Iterative Decoding (BI-STCM-ID) combines bit-interleaved coded modulation (BICM) [1], enjoying both iterative demapping and decoding [2] with the principle of space-time block codes (STBCs) [3], [4]. The amalgamated scheme becomes capable of capturing both space and time diversity and, hence, achieves a high performance under a wide variety of fading conditions.

In practice, the knowledge of the channel impulse response (CIR) is typically acquired using training. However, an excessive number of training symbols may be required, particularly when numerous antennas are involved and/or when the Doppler frequency is high. Hence, precious transmit power, as well as valuable bandwidth, is wasted. Furthermore, a $4 \times 4$ antenna system requires the estimation of 16 channels, for example, which imposes a high complexity in comparison to the idealized coherently detected system benefiting from perfect CIR estimation and, in the presence of realistic estimation, inflicts a substantial performance penalty. Therefore, differentially encoded

Manuscript received July 28, 2006; revised May 25, 2007, July 18, 2007, and July 19, 2007. The review of this paper was coordinated by Prof. L. Lampe.

The authors are with the School of Electronics and Computer Science, University of Southampton, SO17 1BJ Southampton, U.K. (e-mail: nw04r@ ecs.soton.ac.uk; ora02r@ecs.soton.ac.uk; sxn@ecs.soton.ac.uk; lh@ecs.soton. ac.uk).

Color versions of one or more of the figures in this paper are available online at http://ieeexplore.ieee.org.

Digital Object Identifier 10.1109/TVT.2007.907076 low-complexity space-time block coding schemes, dispensing with pilot-based channel estimation and invoking noncoherent detection, have been proposed in [5] and [6].

Another crucial issue in the context of Bit-Interleaved Coded Modulation using Iterative Decoding (BICM-ID) related schemes is the design of constellation mapping, which was studied in [7]. In [8], sphere packing (SP) modulation was proposed to improve the performance of differential space-time block codes (DSTBCs) [6], where the symbols transmitted in two time slots were jointly designed. As a result, the size of the new SP modulated constellation became $L=16$ if originally quadrature phase-shift keying (QPSK) symbols were transmitted. In [9], ten Brink et al. proposed the employment of the so-called Extrinsic Information Transfer (EXIT) characteristics to analyze the convergence behavior of iteratively detected systems, which will be used in our design.

Third, when the channel is classified as nonrecursive, implying that it has a finite-duration impulse response (IR), then the achievable iteration gain of the receiver is limited since there is a limited interleaver gain [10]. However, the channel can be rendered to appear recursive to the receiver, thus resulting in a useful interleaver gain by invoking a recursive inner encoder [11], i.e., a unity-rate precoder. However, there are other methods of rendering the IR duration infinite. For example, in [12], it has been argued that turbo-detected digital phase-shift keying (PSK) has the potential of outperforming coherent PSK, although this requires a number of iterative detection steps. We will demonstrate that the precoder potentially provides a more flexible method of controlling the spread of the extrinsic information using different feedback polynomials and various precoder memory sizes with the aid of EXIT chart analysis [13].

Based on the preceding discussions, in this paper, a precoded SP-aided Bit-Interleaved Differential Space-Time Coded Modulation using Iterative Decoding (BI-DSTCM-ID) design was proposed to improve the achievable performance of BI-STCM-ID schemes. A summary of the novelty and rationale of this paper is presented here.

1) DSTBC is amalgamated with BICM-ID to achieve both spatial and temporal diversity, without the high complexity of channel estimation in coherent detection.

2) The employment of SP modulation allows us to intelligently amalgamate DSTBC and BICM-ID, where we jointly, rather than separately, design the space-time signals of two time slots.

3) Various rate- 1 precoder designs are investigated. The spread of extrinsic information across the transmitted bit stream is governed by the precoder's memory. We will demonstrate that the shape of the EXIT curve, which determines the convergence threshold, is controlled by the precoder's memory.

4) We further improve the performance of the proposed serial concatenated receiver relying on classic convolutional codes by invoking irregular convolutional codes (IRCCs) [14], [15] as an outer code. We jointly consider the flexibility provided by the specifically tailored EXIT characteristics of the IRCCs and the precoder. As a result, we will demonstrate that an even lower $E_{b} / N_{0}$ convergence threshold is attainable in comparison to the system using regular convolutional codes.

The rest of this paper is organized as follows: In Section II, we demonstrate how DSTBC schemes may be designed using SP signal constellations by jointly designing the space-time signals of the individual time slots. Section III provides a detailed description of the encoding and decoding processes of the proposed system. The EXIT chart analysis of the precoded system employing SP modulation using regular/irregular outer channel codes is provided in Section IV. Our 

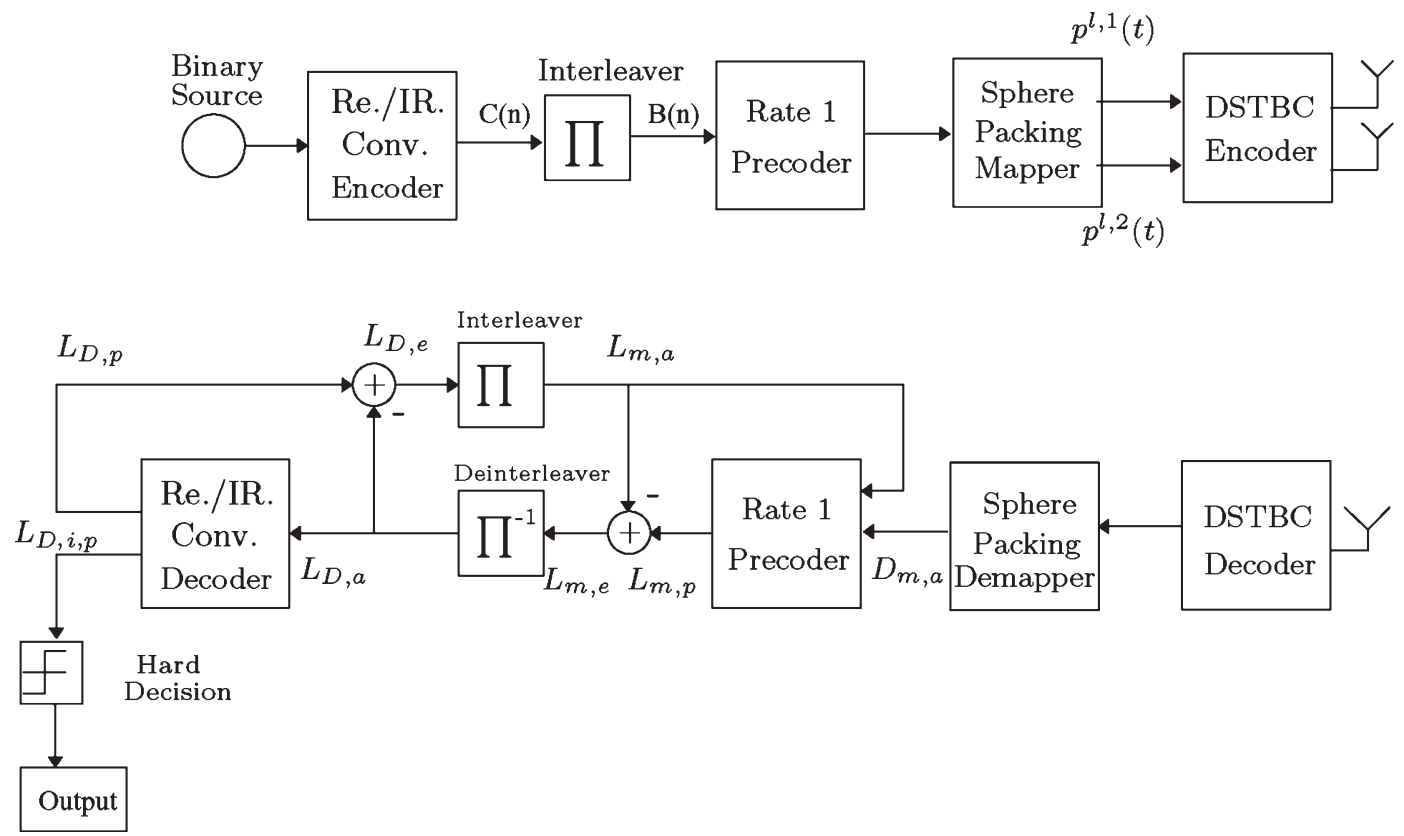

Fig. 1. Schematic of the precoded SP-aided BI-DSTCM-ID design using regular/irregular convolution codes.

simulation results are discussed in Section V. Finally, we conclude in Section VI.

\section{ORThogonal Design With SP Modulation}

Orthogonal transmit diversity designs can be recursively described [16] as follows: Let $G_{1}\left(x^{1}\right)=x^{1} I_{1}$, and

$$
G_{2^{k}}\left(x^{1}, \ldots, x^{k+1}\right)=\left[\begin{array}{cc}
G_{2^{k-1}}\left(x^{1}, \ldots, x^{k}\right) & x^{k+1} I_{2^{k-1}} \\
-\left(x^{k+1}\right)^{*} I_{2^{k-1}} & G_{2^{k-1}}^{\mathcal{H}}\left(x^{1}, \ldots, x^{k}\right)
\end{array}\right]
$$

for $k=1,2,3, \ldots$, where ${ }^{*}$ denotes the complex conjugate, ${ }^{\mathcal{H}}$ denotes the Hermitian transpose, and $I_{2^{k-1}}$ is a $2^{k-1} \times 2^{k-1}$ identity matrix. Then, $G_{2^{k}}\left(x^{1}, x^{2}, \ldots, x^{k+1}\right)$ constitutes an orthogonal design of size $2^{k} \times 2^{k}$, which maps the complex variables representing $x^{1}, x^{2}, \ldots, x^{k+1}$ to $2^{k}$ transmit antennas.

It was shown in [17] that the diversity product quantifying the coding advantage of an orthogonal transmit diversity scheme is determined by the minimum Euclidean distance of vectors $x^{1}$, $x^{2}, \ldots, x^{k+1}$. Therefore, it was proposed in [17] to use SP schemes that have the best-known minimum Euclidean distance in the $2(k+1)$-dimensional real-valued Euclidean space $R^{2(k+1)}$.

In this contribution, differential space-time systems [18] obeying the structure of Fig. 1 and employing two transmit antennas are considered. It was shown in [18] that, when the received signals are differentially decoded, the resultant signals will be scaled versions of transmitted symbols $p^{1}(t)$ and $p^{2}(t)$, which are corrupted by complex additive white Gaussian noise (AWGN) similar to the coherent $G_{2}$ STBC of [3]. This observation implies that the diversity product of differential STBC systems is determined by the minimum Euclidean distance of all legitimate vectors $\left(p^{1}, p^{2}\right)$, where the time index is removed for notational simplicity.

For the sake of generalizing our treatment, let us assume that there are $L$ legitimate twin-time-slot vectors $\left(p^{l, 1}, p^{l, 2}\right), l=0,1, \ldots$, $L-1$, where $L$ represents the number of SP modulated symbols. The encoder, then, has to choose the modulated symbol associated with each block of bits from these $L$ legitimate symbols. In contrast to the independent DSTBC signaling pulses $p^{l, 1}$ and $p^{l, 2}$, our aim is to design $p^{l, 1}$ and $p^{l, 2}$ jointly, so that they have the best minimum Euclidean distance from all other $(L-1)$ legitimate SP symbols since this minimizes the system's SP symbol error probability.

Let $a_{l, 1}, a_{l, 2}, a_{l, 3}, a_{l, 4}, l=0,1, \ldots, L-1$ be the SP phasor points selected from the 4-D real-valued Euclidean space $\mathrm{R}^{4}$, where each of the four elements $a_{l, 1}, a_{l, 2}, a_{l, 3}$, and $a_{l, 4}$ gives one coordinate of the twin-time-slot complex-valued phasor points. Hence, $p^{l, 1}$ and $p^{l, 2}$ can be written as

$$
\begin{aligned}
\left\{p^{l, 1}, p^{l, 2}\right\} & =T\left(a_{l, 1}, a_{l, 2}, a_{l, 3}, a_{l, 4}\right) \\
& =\left\{a_{l, 1}+j a_{l, 2}, a_{l, 3}+j a_{l, 4}\right\}
\end{aligned}
$$

In the 4-D real-valued Euclidean space $\mathrm{R}^{4}$, the lattice $D_{4}$ is defined as an SP having the best minimum Euclidean distance from all other $(L-1)$ legitimate SP constellation points in $\mathrm{R}^{4}$ [19]. More specifically, $D_{4}$ may be defined as a lattice that consists of all legitimate SP constellation points having integer coordinates $\left[\begin{array}{llll}a_{1} & a_{2} & a_{3} & a_{4}\end{array}\right]$ that uniquely and unambiguously describe the legitimate combinations of the DSTBC modulated symbols $p^{l, 1}$ and $p^{l, 2}$ of each time slot but is subjected to the SP constraint of $a_{1}+a_{2}+a_{3}+a_{4}=K$, where $K$ is an even integer. Let us assume that $Q=\left\{q^{l}=\left[a_{l, 1}, a_{l, 2}, a_{l, 3}, a_{l, 4}\right] \in\right.$ $\left.\mathrm{R}^{4}: 0 \leq l \leq L-1\right\}$ constitutes a set of $L$ legitimate constellation points selected from lattice $D_{4}$ having a total energy of $E \triangleq$ $\sum_{l=0}^{L-1}\left(\left|a_{l, 1}\right|^{2}+\left|a_{l, 2}\right|^{2}+\left|a_{l, 3}\right|^{2}+\left|a_{l, 4}\right|^{2}\right)$ and introduce the set of complex constellation symbols $\left\{C_{l}: 0 \leq l \leq L-1\right\}$ given by

$$
C_{l}=\sqrt{\frac{2 L}{E}}\left(p^{l, 1}, p^{l, 2}\right), \quad l=0,1, \ldots, L-1
$$

whose diversity product is determined by the minimum Euclidean distance of the set of $L$ legitimate constellation points in $Q$. For example, at the first layer of the SP lattice, there are 24 constellation points according to all the possible constellations of the values $[ \pm 1$; $\pm 1,0,0]$, and the constellation set of $L=16$ points was found by computer search upon maximizing their Euclidean distance at a given total power $E$. 


\section{SYSTEM DESCRIPTION}

We consider a wireless communication system invoking multiple transmit antennas at the base station and an arbitrary number of receive antennas at the mobile station. The block diagram of the system is shown in Fig. 1.

At time instant $t$, a frame of $n$ information bits is encoded by a regular/irregular outer half-rate convolutional encoder. Then, the encoded bits $C(n)$ of Fig. 1 are permuted by a random interleaver $\pi$. The interleaved bits $B(n)$ are then encoded by a rate- 1 recursive precoder, which contributes toward achieving a high iteration gain, as will be demonstrated in Section V. Then, the SP mapper of Fig. 1 maps each block of $b$ convolutionally encoded bits to a legitimate constellation point $q^{l} \in Q$ of the SP lattice $D_{4}$, where we have $b=$ $\log _{2} L$. Note that optimal mapping is chosen based on the analysis of EXIT. Further detail can be found in [8], as we will demonstrate in Section IV. The same mapper then represents the constellation point $q^{l}$ by two complex DSTBC symbols $p^{l, 1}(t)$ and $p^{l, 2}(t)$ of the two time slots using (2) and (3). Subsequently, the DSTBC encoder of Fig. 1 calculates the symbols to be transmitted as follows:

$$
\begin{aligned}
\left(x_{2 t+1}^{1}, x_{2 t+1}^{2}\right)=\triangle p^{l, 1}(t)\left(x_{2 t-1}^{1},\right. & \left.x_{2 t-1}^{2}\right) \\
& +\triangle p^{l, 2}(t)\left(-x_{2 t-1}^{2 *}, x_{(2 t-1}^{1 *}\right)
\end{aligned}
$$

where we have $\triangle=1 / \sqrt{\left|x^{1}(t-1)\right|^{2}+\left|x^{2}(t-1)\right|^{2}}$. Then, the differentially encoded symbols are transmitted using the $G_{2}$ matrix of (1). Furthermore, instead of directly transmitting the differentially encoded symbols, the proposed system may also be amalgamated with differential space-time spreading (STS) [20]. Since each spreading sequence of the STS scheme spans two symbol periods while transmitting two symbols, which is identical to the philosophy of DSTBC, it can be argued that the employment of STS allows the system to accommodate two users without affecting the achievable single-user throughput. Similarly, multiple users may be supported with the aid of multiple antennas and longer spreading sequences.

The structure of the receiver is shown in Fig. 1, where the received signal at instant $2 t+1$ and $2 t+2$ is given by

$$
\begin{aligned}
& r_{2 t+1}=h_{1}(2 t+1) x_{2 t+1}^{1}+h_{2}(2 t+1) x_{2 t+1}^{2}+n_{2 t+1} \\
& r_{2 t+2}=-h_{1}(2 t+1) x_{2 t+1}^{2 *}+h_{2}(2 t+1) x_{2 t+1}^{1 *}+n_{2 t+2} .
\end{aligned}
$$

The CIR $h_{m}(t)$, for $m=1,2$, corresponding to the $m$ th transmit antenna, is modeled as a correlated Rayleigh fading channel, and its magnitude and phase are assumed to be constant for two consecutive DSTBC transmission blocks. The rate of fading is governed by the normalized Doppler frequency $f_{d}$. We assume that the fading experienced by all diversity channels is independent, whereas $n(t)$ is the corresponding complex-valued AWGN. Then, the conventional DSTBC decoder can recover the transmitted symbols by calculating

$$
\begin{aligned}
\tilde{x}_{2 t+1}^{1} & =r_{2 t+1} r x_{2 t-1}^{*}+r_{2 t} r_{2 t+2}^{*} \\
& =\left(\left|h_{1}(2 t+1)\right|^{2}+\left|h_{2}(2 t+1)\right|^{2}\right) x_{2 t+1}^{1}+\tilde{N}_{1} \\
\tilde{x}_{2 t+1}^{2} & =r_{2 t+1} r_{2 t}^{*}-r_{2 t-1} r_{2 t+2}^{*} \\
& =\left(\left|h_{1}(2 t+1)\right|^{2}+\left|h_{2}(2 t+1)\right|^{2}\right) x_{2 t+1}^{2}+\tilde{N}_{2}
\end{aligned}
$$

where $\tilde{N}_{1}$ and $\tilde{N}_{2}$ are the diversity combined noise vectors.

Then, the decoded symbols of (7) are passed to the SP demapper, where they are demapped to their log-likelihood ratio (LLR) representation for each of the convolutional coded bits in an SP symbol $D_{m, a}$. The rate-1 precoder's decoder processes the a priori information $L_{m, a}$ fed back from the outer decoder and the output $D_{m, a}$ of the SP
TABLE I

SYSTEM PARAMETERS

\begin{tabular}{|l|r|}
\hline Modulation & sphere packing $L=16$ \\
\hline Mapping & Gray mapping \\
\hline Number of Transmitters & 2 \\
\hline Number of Receivers & 1 \\
\hline Normalised Doppler Frequency & $f_{d}=0.01$ \\
\hline Regular Outer Channel Code & RSC $(2,1,5)$ \\
& $\left(G_{r}, G\right)=(35,23)_{8}$ \\
\hline IRCC & Half-rate \\
\hline Interleaver Length & $10^{6}$ bits \\
\hline Precoder Rate & 1 \\
\hline Precoder Memory & 1 \\
\hline Throughput & 8 \\
\hline Spreading Factor & 1 bit per channel use \\
\hline
\end{tabular}

demapper of Fig. 1 . The a priori LLR values $L_{m, a}$ of the rate-1 precoder are subtracted from the a posteriori LLR values $L_{m, p}$ for the sake of generating the extrinsic LLR values $L_{m, e}$. Then, the LLRs $L_{m, e}$ are deinterleaved by a soft-bit interleaver to generate $L_{D, a}$, as shown in Fig. 1. Next, the soft bits $L_{D, a}$ are passed to the convolutional decoder to compute the a posteriori LLR values $L_{D, p}$ provided by the $\log$ maximum a posteriori algorithm [21] for all the channel coded bits. During the last iteration, only the LLR values of $L_{D, i, p}$ are subjected to hard decision. The extrinsic feedback information $L_{D, e}$ of Fig. 1 is generated by subtracting the a priori information from the a posteriori information according to $L_{D, p}-L_{D, a}$. Observe in Fig. 1 that $L_{m, a}$ is the interleaved version of $L_{D, e}$. The precoder's decoder exploits the a priori information for the sake of providing improved a posteriori LLR values, which are then passed to the channel decoder of Fig. 1 and, in turn, back to the precoder's decoder for further iterations. More detailed discussions on the iterative decoding process are provided in [22].

\section{EXIT Chart ANALYSis}

The application of EXIT charts [13] in the design of our serially concatenated system facilitates the prediction of its convergence behavior, based on the extrinsic information exchange between the inner and outer coders. The accuracy of EXIT charts is based on two assumptions. First, we assume that the a priori LLR values become fairly uncorrelated when employing a high interleaver length. Second, we assume that the probability density function of the a priori LLR values is Gaussian. In this section, a system using two transmit and one receive antenna, as well as the parameters listed in Table I, is considered.

More explicitly, for the inner decoder, the mutual information of $I_{A_{m}}=I\left(B(n), L_{m, a}\right), 0 \leq I_{A_{m}} \leq 1$ between the outer coded bits $B(n)$ of Fig. 1 and the LLR values of $L_{m, a}$ is used to quantify the information content of the a priori knowledge at the input of the inner decoder. Here, we consider the serially concatenated precoder, SP mapper, and DSTBC encoder as the amalgamated inner encoder. By contrast, to quantify the information content of the extrinsic information of $L_{m, e}$ at the output of the inner encoder shown in Fig. 1, the mutual information $I_{E_{m}}=I\left(B(n), L_{m, e}\right)$ can be used. Considering $I_{E_{m}}$ as a function of both $I_{A_{m}}$ and the $E_{b} / N_{0}$ value, the inner code's EXIT characteristic is defined as $I_{E_{m}}=T_{M}\left(I_{A_{m}}, E_{b} / N_{0}\right)$ [13].

The extrinsic transfer characteristic of the outer channel decoder describes the relationship between the outer channel coded input $L_{D, a}$ and the outer channel decoded extrinsic output $L_{D, e}$. Therefore, the EXIT characteristic of the outer channel decoder is independent of the $E_{b} / N_{0}$ value and, hence, can be written as $I_{E_{D}}=$ $T_{D}\left(I_{A_{D}}\right)$, where $I_{A_{D}}=I\left(C(n), L_{D, a}\right), 0 \leq I_{A_{D}} \leq 1$ is the mutual 


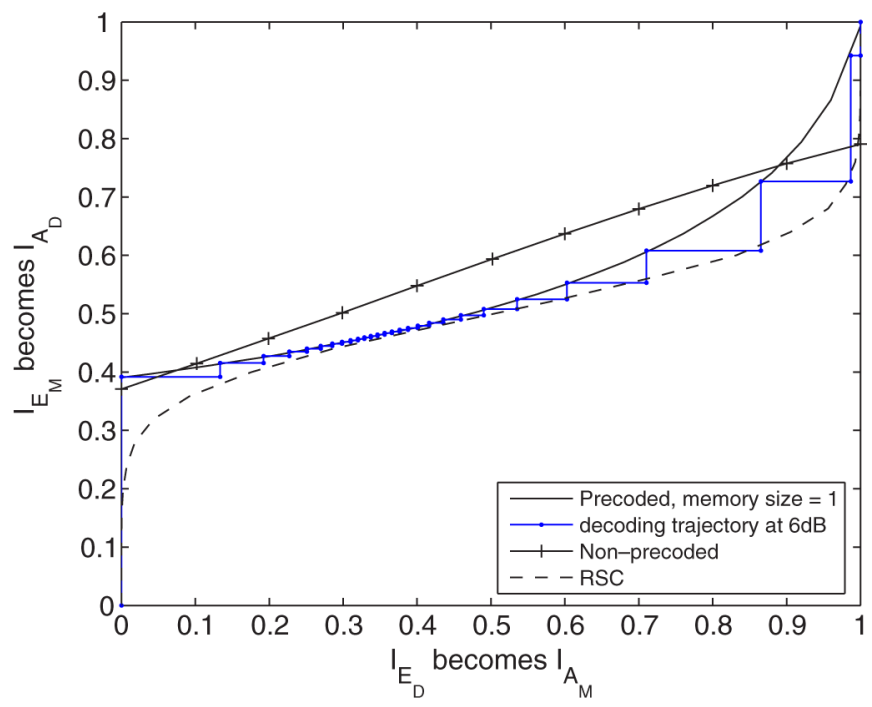

Fig. 2. EXIT chart comparison of non-precoded and memory-size-1 precoded systems at $E_{b} / N_{0}=6 \mathrm{~dB}$ when using the system parameters outlined in Table I and actual decoding trajectory.

information between the outer channel coded bits $C(n)$ and the LLR values $L_{D, a}$. Similarly, $I_{E_{D}}=I\left(C(n), L_{D, e}\right), 0 \leq I_{E_{D}} \leq 1$ is the mutual information between the outer channel coded bits $C(n)$ and the LLR values $L_{D, e}$.

The EXIT chart of the precoded system of Fig. 1 is shown in Fig. 2, when using a memory-1 precoder, together with the recursive systematic convolutional (RSC) code and the system parameters outlined in Table I. This scheme is compared to a non-precoded system when operating at $E_{b} / N_{0}=6 \mathrm{~dB}$. The non-precoded system also employs iterative decoding between the soft SP demapper and the outer channel decoder, as described in [23] and [24], where the best anti-Gray mapping scheme that matches the outer code's EXIT curve was chosen as in [8]. Ideally, in order for the extrinsic information exchange between the inner and outer codes to converge at a certain $E_{b} / N_{0}$ value, the extrinsic transfer curves should only intersect at the $\left(I_{A_{M}}, I_{E_{M}}\right)=(1.0,1.0)$ point. If this condition is satisfied, then a socalled convergence tunnel [13] appears in the EXIT chart. Even if there is no open tunnel in the EXIT chart leading to point $(1.0,1.0)$ but the two curves intersect at a point close to $I_{E_{M}}=1.0$, then a sufficiently low bit error rate (BER) may still be achievable.

Observe in Fig. 2 that the precoded system reaches point $I_{E} \approx$ 0.998 , where a convergence tunnel has taken shape, hence resulting in an infinitesimally low BER. By contrast, the non-precoded scheme reaches $I_{E_{M}} \approx 0.79$, as shown in Fig. 2; hence, only a modest BER advantage can be achieved. In other words, it is expected that the precoded system is capable of reaching the typical turbo cliff, when considering the BER versus $E_{b} / N_{0}$ performance, as will be shown in Fig. 6.

Fig. 3 shows the EXIT curves of the inner decoder operating at $E_{b} / N_{0}=6 \mathrm{~dB}$, when using various precoder memory sizes. When the memory size of the precoder is increased, the EXIT curves of the inner decoder tend to start at lower $I_{E_{M}}$ values but rise more rapidly. For $E_{b} / N_{0}=6 \mathrm{~dB}$, a memory-1 precoded system has an open convergence tunnel, whereas a higher precoder memory tends to require a higher $E_{b} / N_{0}$ value for an open convergence tunnel to take shape. Hence, for this particular system employing an $\operatorname{RSC}(2,1,5)$ outer code, a precoder memory size of 1 is capable of achieving an infinitesimally low BER at the lowest possible $E_{b} / N_{0}$ value. Note that the area under all the EXIT curves recorded for various precoder memory sizes is constant, which indicates that the achievable channel

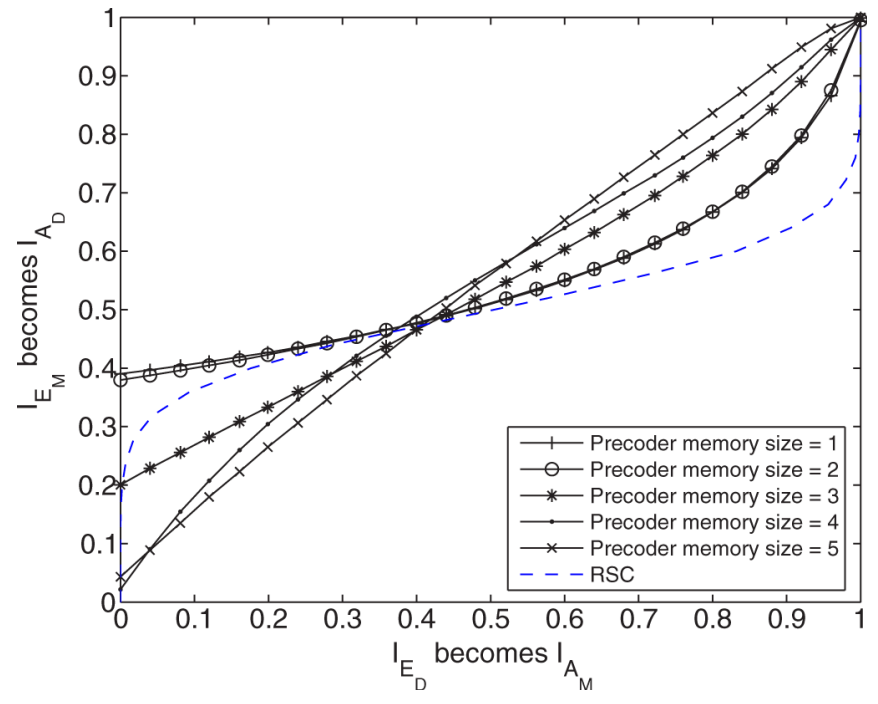

Fig. 3. Inner code EXIT characteristics using Gray mapping for different precoder memory sizes when employing the system parameters outlined in Table I and operating at $E_{b} / N_{0}=6 \mathrm{~dB}$.

capacity is not affected [25], [26] by changing the shape of the EXIT curves.

The open EXIT tunnel area between the inner and outer decoders' curves in Fig. 2 is not necessarily an unambiguous characteristic of the system since the convergence tunnel is only narrow within a certain range. By shaping both the inner and outer EXIT curves, it is potentially possible to create an even narrower tunnel in conjunction with a lower $E_{b} / N_{0}$ requirement. Since IRCCs exhibit flexible EXIT characteristics, we adopt an IRCC as our outer code while keeping the overall code rate the same as that of our previous RSC code. An IRCC scheme constituted by a set of $P=17$ subcodes was constructed in [15] from a systematic half-rate memory- 4 mother code defined by the octally represented generator polynomials $(23,35)_{8}$. Each subcode encodes a specific fraction of the incoming bit stream, as quantified by the weighting coefficients $\alpha_{i}, i=1,2, \ldots, 17$. Hence, $\alpha_{i}$ is optimized with the aid of the iterative algorithm of [14], so that the EXIT curve of the resultant IRCC closely matches that of the inner code. Since the inner code's EXIT curve benefits from the flexibility provided by the precoder's memory size, as shown in Fig. 3, the IRCC is optimized for all the different memory sizes featured in Fig. 3. Explicitly, our design objective is to find the lowest $E_{b} / N_{0}$ value, where it is possible to form an open convergence tunnel.

Fig. 4 illustrates both the EXIT curves of the IRCC optimized for the proposed system having a precoder memory size of 3 as well as the bit-by-bit decoding trajectory at $E_{b} / N_{0}=5.5 \mathrm{~dB}$, where the optimized weighting coefficients are given as follows: $\left[\alpha_{1}, \ldots, \alpha_{17}\right]=$ $[0,0,0.0206,0.0199,0.1567,0,0,0.0811,0.1803,0.0835,0,0$, $0.2757,0,0.0302,0.0326,0.1194]$. Observe that an extremely narrow EXIT tunnel is formed, which exhibits a $0.5-\mathrm{dB}$ advantage over the RSC-coded schemes.

Finally, the benefits of employing SP modulation over conventional modulation schemes recorded for the iteratively detected schemes are characterized in Fig. 5, where SP modulation employing $L=16$ constellation points and various mapping schemes is compared to classic QPSK at $E_{b} / N_{0}=6 \mathrm{~dB}$. Note that SP using Gray labeling is capable of generating higher extrinsic information than various anti-Gray mapping schemes since there is no information exchange between the SP demapper and the precoder's decoder [27]. Since SP-aided DSTBC facilitated the joint design of the space-time dimension available, more extrinsic information was obtained with the aid of 


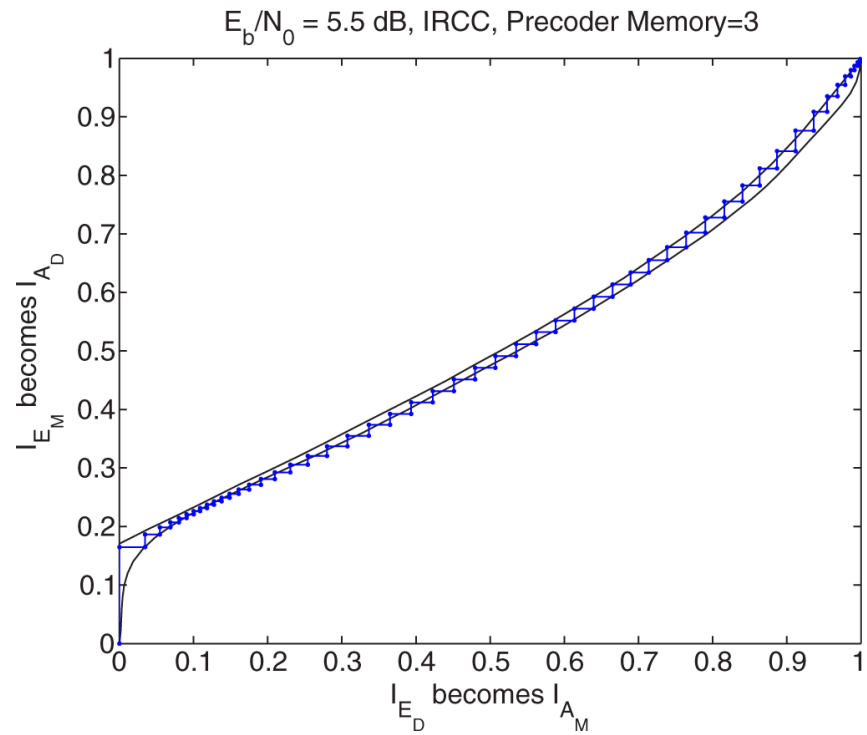

Fig. 4. EXIT chart of memory-size-3 precoded systems using IRCC at $E_{b} / N_{0}=5.5 \mathrm{~dB}$ when using the system parameters outlined in Table I and the actual decoding trajectory at $E_{b} / N_{0}=5.5 \mathrm{~dB}$.

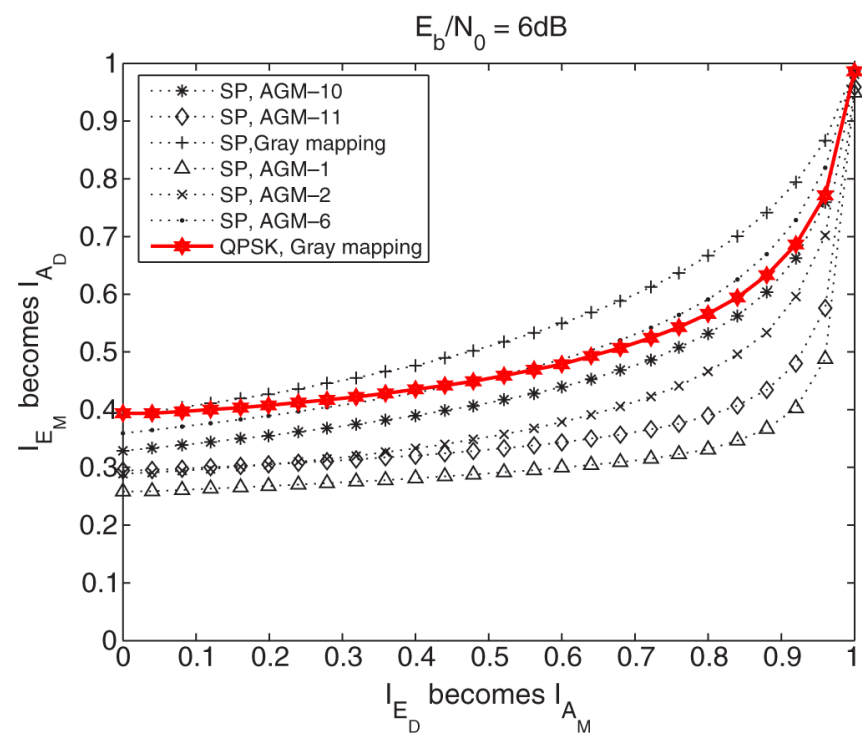

Fig. 5. EXIT comparison of system employing SP using various mappings and QPSK with Gray mapping when employing the system parameters outlined in Table I.

the precoder compared to conventional QPSK modulation. Therefore, the proposed SP-aided scheme using Gray labeling exhibited a larger area under the EXIT curve compared to its QPSK counterpart, as shown in Fig. 5.

\section{Simulation Result and Discussion}

We now proceed to characterize the achievable BER performance of the turbo-detected precoded SP-aided BI-DSTCM-ID system of Fig. 1. All the simulation parameters are listed in Table I, unless otherwise specified.

Fig. 6 demonstrates the BER performance of the turbo-detected precoded system, and a non-precoded scheme is characterized as a benchmarker, where iterative detection is carried out by exchanging extrinsic information between the SP demapper and the outer channel

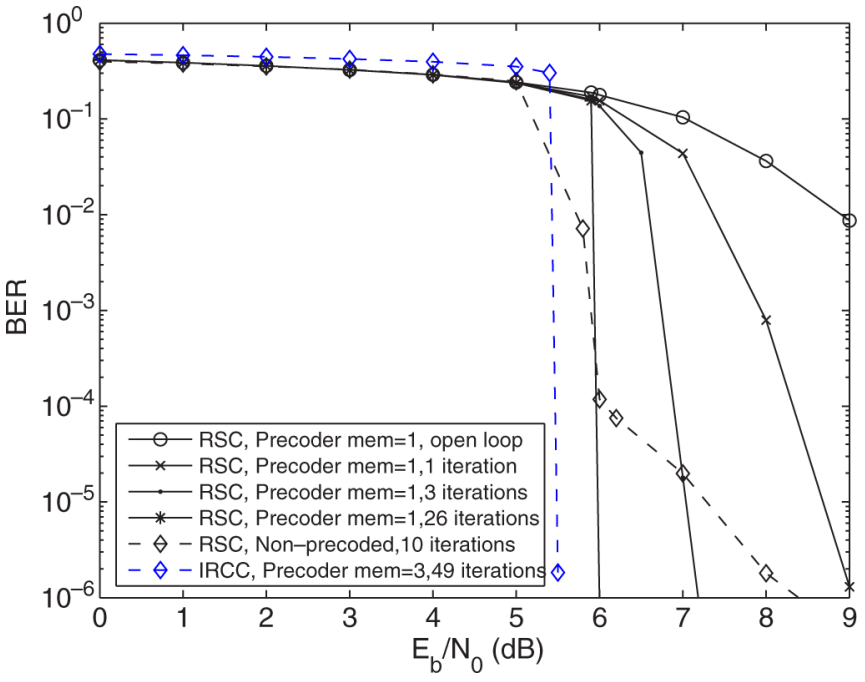

Fig. 6. BER performance of a half-rate RSC/IRCC-coded SP-aided BI-DSTCM-ID system both with and without precoder having a memory size of 1 or 3 when employing the system parameters outlined in Table I.

decoder. The precoding-aided RSC-coded system exhibits a turbo cliff at $E_{b} / N_{0}=6 \mathrm{~dB}$, which is about $0.2 \mathrm{~dB}$ higher than that of the non-precoded system. However, the precoded system is capable of achieving an infinitesimally low BER since its convergence tunnel leads to the $\left(I_{A_{M}}, I_{E_{M}}\right)=(1.0,1.0)$ point. This observation matches the prediction of the EXIT charts shown in Fig. 2. At BER $=10^{-6}$, the precoding advantage is about $3.4 \mathrm{~dB}$. Note that the BER advantage of the precoded schemes is achieved at the price of a higher decoding complexity since $I=26$ iterations are required to reach the turbo-cliff region, whereas only $I=10$ iterations are required by the non-precoded scheme.

Furthermore, the BER performance of the IRCC-coded scheme using a precoder memory size of 3 is also shown in Fig. 6. As predicted in Fig. 4, the system achieved an infinitesimally low BER at $E_{b} / N_{0}=$ $5.5 \mathrm{~dB}$, where a 0.5 -dB signal-to-noise ratio gain is observed over the RSC-coded scheme having a precoder memory size of 1 . Naturally, a higher number of decoding iterations is needed in addition to the increased decoding complexity imposed by the precoder memory size of 3 .

\section{CONCLUSiON}

In this paper, we have investigated a precoded SP-aided BI-DSTCM-ID system that exploits the combined advantages of both precoding and iterative decoding. EXIT chart analysis was employed to study the convergence behavior of the proposed system. Our investigations demonstrated that the precoded systems outperform their non-precoded counterparts in the low-BER region. However, there will be no advantage in terms of BER reduction when increasing the precoder's memory size if a regular convolutional code was employed. We also note that the employment IRCCs, together with the flexibility of the precoder's memory size, facilitates the design of turbo-detected systems converging at low $E_{b} / N_{0}$ values.

\section{REFERENCES}

[1] G. Caire, G. Taricco, and E. Biglieri, "Bit-interleaved coded modulation," IEEE Trans. Inf. Theory, vol. 44, no. 3, pp. 927-946, May 1998.

[2] X. Li and J. Ritcey, "Bit-interleaved coded modulation with iterative decoding using soft feedback," Electron. Lett., vol. 34, no. 10, pp. 942-943, May 1998. 
[3] S. M. Alamouti, "A simple transmit diversity technique for wireless communications," IEEE J. Sel. Areas Commun., vol. 16, no. 8, pp. 1451-1458, Oct. 1998.

[4] V. Tarokh, H. Jafarkhani, and A. R. Calderbank, "Space-time block coding for wireless communications: Performance results," IEEE J. Sel. Areas Commun., vol. 17, no. 3, pp. 451-460, Mar. 1999.

[5] B. M. Hochwald and W. Sweldens, "Differential unitary space-time modulation," IEEE Trans. Commun., vol. 48, no. 12, pp. 2041-2052, Dec. 2000.

[6] V. Tarokh and H. Jafarkhani, "A differential detection scheme for transmit diversity," IEEE J. Sel. Areas Commun., vol. 18, no. 7, pp. 1169-1174, Jul. 2000.

[7] Y. Li and X.-G. Xia, "Constellation mapping for space-time matrix modulation with iterative demodulation/decoding," IEEE Trans. Commun., vol. 53, no. 5, pp. 764-768, May 2005.

[8] O. Alamri, N. Wu, and L. Hanzo, "A differential turbo detection aided sphere packing modulated space-time coding scheme," in Proc. IEEE Veh. Technol. Conf.-Spring, 2006, vol. 5, pp. 2474-2478.

[9] S. ten Brink, J. Speidel, and R.-H. Yan, "Iterative demapping and decoding for multilevel modulation," in Proc. IEEE GLOBECOM Conf., Nov. 1998, vol. 1, pp. 579-584.

[10] K. Narayanan and G. Stüber, "A serial concatenation approach to iterative demodulation and decoding," IEEE Trans. Commun., vol. 47, no. 7, pp. 956-961, Jul. 1999.

[11] D. Divsalar, S. Dolinar, and F. Pollara, "Serial concatenated trellis coded modulation with rate-1 inner code," in Proc. IEEE GLOBECOM Conf., 2000, vol. 2, pp. 777-782.

[12] P. Hoeher and J. Lodge, "Turbo DPSK: Iterative differential PSK demodulation and channel decoding," IEEE Trans. Commun., vol. 47, no. 6, pp. 837-843, Jun. 1999.

[13] S. ten Brink, "Designing iterative decoding schemes with the extrinsic information transfer chart," AEÜ Int. J. Electron. Commun., vol. 54, no. 6, pp. 389-398, 2000.

[14] M. Tüchler and J. Hagenauer, "Exit charts of irregular codes," in Proc. 36th CISS, 2002, pp. 748-753.

[15] M. Tüchler, "Design of serially concatenated systems depending on the block length," IEEE Trans. Commun., vol. 52, no. 2, pp. 209-218, Feb. 2004

[16] W. Su and X.-G. Xia, "On space-time block codes from complex orthogonal designs," Wireless Pers. Commun., vol. 25, no. 1, pp. 1-26, Apr. 2003.

[17] W. Su, Z. Safar, and K. J. R. Liu, "Space-time signal design for timecorrelated Rayleigh fading channels," in Proc. IEEE ICC, 2003, vol. 5, pp. 3175-3179.

[18] C. Hwang, S. Nam, J. Chung, and V. Tarokh, "Differential space time block codes using nonconstant modulus constellations," IEEE Trans. Signal Process., vol. 51, no. 11, pp. 2955-2964, Nov. 2003.

[19] J. H. Conway and N. J. A. Sloane, Sphere Packings, Lattices and Groups. New York: Springer-Verlag, 1998.

[20] B. M. Hochwald, T. Marzetta, and C. Papadias, "A transmitter diversity scheme for wideband CDMA systems based on space-time spreading," IEEE J. Sel. Areas Commun., vol. 19, no. 1, pp. 48-60, Jan. 2001.

[21] P. Robertson, E. Villebrun, and P. Hoeher, "A comparison of optimal and sub-optimal MAP decoding algorithms operating in the log domain," in Proc. IEEE ICC, 1995, vol. 2, pp. 1009-1013.

[22] S. Benedetto, D. Divsalar, G. Montorsi, and F. Pollara, "Serial concatenation of interleaved codes: Performance analysis, design, and iterative decoding," IEEE Trans. Inf. Theory, vol. 44, no. 3, pp. 909-926, May 1998.

[23] O. R. Alamri, B. L. Yeap, and L. Hanzo, "A turbo detection and spherepacking-modulation-aided space-time coding scheme," IEEE Trans. Veh. Technol., vol. 56, no. 2, pp. 575-582, Mar. 2007.

[24] L. Hanzo, T. H. Liew, and B. L. Yeap, Turbo Coding, Turbo Equalisation, and Space-Time Coding for Transmission Over Fading Channels. Hoboken, NJ: Wiley, 2002.

[25] A. Ashikhmin, G. Kramer, and S. ten Brink, "Code rate and the area under extrinsic information transfer curves," in Proc. IEEE Int. Symp. Inf. Theory, 2002, p. 115.

[26] A. Ashikhmin, G. Kramer, and S. ten Brink, "Extrinsic information transfer functions: Model and erasure channel properties," IEEE Trans. Inf. Theory, vol. 50, no. 11, pp. 2657-2673, Nov. 2004.

[27] Y. Huang and J. A. Ritcey, "Optimal constellation labeling for iteratively decoded bit-interleaved space-time coded modulation," IEEE Trans. Inf. Theory, vol. 51, no. 5, pp. 1865-1871, May 2005.

\section{A Suboptimal Maximum-Likelihood Receiver for FFH/BFSK Systems With Multitone Jamming Over Frequency-Selective Rayleigh-Fading Channels}

\author{
Tsan-Ming Wu, Member, IEEE
}

\begin{abstract}
Maximum-likelihood (ML) receivers have been investigated for the fast frequency-hopped binary frequency-shift-keying (FFH/BFSK) spread-spectrum communications system with the worst case of the band multitone jamming and the additive white Gaussian noise over frequencyselective Rayleigh-fading channels. A suboptimal ML diversity-combining receiver has been theoretically analyzed based upon an approximation of the natural logarithm of the modified Bessel function under a correlated two-path frequency-selective channel model. The performance of the receiver compared with that of other diversity-combining receivers, in terms of the bit error rate, has been scrutinized. Computer simulations confirm that our numerical analysis is valid, and the proposed suboptimal ML receiver outperforms other receivers.
\end{abstract}

Index Terms-Fast frequency-hopped binary frequency-shift keying (FFH/BFSK), frequency-selective fading channel, maximum-likelihood (ML) receiver, multitone jamming (MTJ).

\section{INTRODUCTION}

Frequency-hopped (FH) spread-spectrum systems have been widely used in military and commercial applications for mitigating the impacts of the deep fades and various types of jamming. Conventionally, fast frequency hopping (FFH) with a noncoherent frequency-shiftkeying modulation has been used to protect the transmitting signals against a certain hostile jammer. Multitone jamming (MTJ) and partial-band noise jamming (PBNJ) are two principal categories of interference models. However, under the same total jamming power, the MTJ strategy causes more critical harm than the PBNJ scheme does, because the former possesses more effective power utilization. Consequently, various diversity-combining approaches have been extensively studied against the MTJ impacts over frequency-nonselective fading channels such as product-combining [1], self-normalizing [2], ratio-statistic-combining [3], and maximum-likelihood (ML) diversity-combining [4], [5] receivers. Among them, the ML diversitycombining receiver under the MTJ circumstance has been studied in [4], although it was first investigated in [6] under the PBNJ environment. Han and Teh [5] further proposed two suboptimum ML diversity-combining schemes for the $\mathrm{FFH} / m$-ary frequency-shiftkeying system with MTJ over frequency-nonselective fading channels and proved that those algorithms significantly outperform other diversity-combining counterparts. However, the ML diversitycombining receiver with MTJ over frequency-selective fading channels has yet to be analyzed. In this correspondence, we will investigate

Manuscript received October 12, 2006; revised January 23, 2007, April 26, 2007, and June 14, 2007. This paper was presented in part at the IEEE International Conference on Communications (ICC'2007), Glasgow, U.K. The review of this paper was coordinated by Dr. A. Ghrayeb.

The author is with the Department of Electrical Engineering, Chung Yuan Christian University, Chung-Li 32023, Taiwan, R.O.C. (e-mail: tmw@ dec.ee.cycu.edu.tw).

Color versions of one or more of the figures in this paper are available online at http://ieeexplore.ieee.org.

Digital Object Identifier 10.1109/TVT.2007.905623 